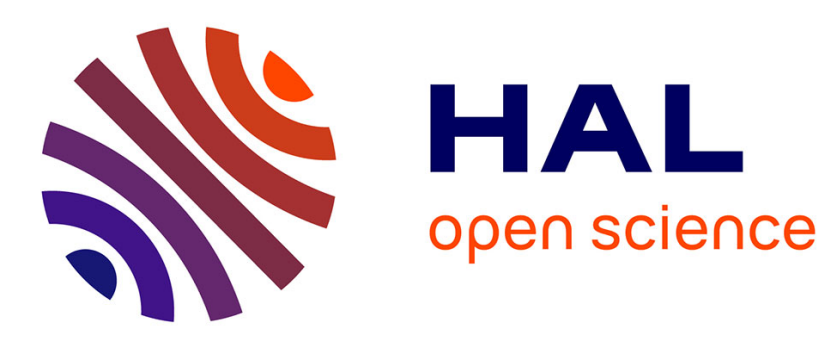

\title{
If I don't know, should I infer? Reasoning around ignorance in a many-valued framework
}

Adrien Revault d'Allonnes, Marie-Jeanne Lesot

\section{To cite this version:}

Adrien Revault d'Allonnes, Marie-Jeanne Lesot. If I don't know, should I infer? Reasoning around ignorance in a many-valued framework. 17th World Congress of the International Fuzzy Systems Association, IFSA2017, Jun 2017, Otsu, Japan. hal-01540164

\section{HAL Id: hal-01540164 https://hal.sorbonne-universite.fr/hal-01540164}

Submitted on 5 Oct 2017

HAL is a multi-disciplinary open access archive for the deposit and dissemination of scientific research documents, whether they are published or not. The documents may come from teaching and research institutions in France or abroad, or from public or private research centers.
L'archive ouverte pluridisciplinaire HAL, est destinée au dépôt et à la diffusion de documents scientifiques de niveau recherche, publiés ou non, émanant des établissements d'enseignement et de recherche français ou étrangers, des laboratoires publics ou privés. 


\section{If I don't know, should I infer? Reasoning around ignorance in a many-valued framework}

\author{
Adrien Revault d'Allonnes \\ Université Paris 8, EA 4383, LIASD, \\ FR-93526, Saint-Denis, France \\ Email: Allonnes@ai.univ-paris8.fr
}

\author{
Marie-Jeanne Lesot \\ Sorbonne Universités, LIP6, \\ UPMC Univ Paris 06, CNRS, UMR 7606 \\ F-75005, Paris, France \\ Email: Marie-Jeanne.Lesot@lip6.fr
}

\begin{abstract}
Many-valued logic allows to reason with partial truth measured by degrees on a discrete scale, but it suffers from an ambiguous interpretation of the middle truth level, considered as intermediate truth or as ignorance, i.e. inability to assess truth. The $\mathcal{L}_{M}^{e}$ extension introduces an additional value, outside the truth scale, to distinguish between these two notions. This paper studies $\mathcal{L}_{M}^{e}$ from a logical perspective, examining how to reason in this framework: it discusses the definition of appropriate semantics for the logical connectives and it considers an inference task, proposing a Modus Ponens variant for $\mathcal{L}_{M}^{e}$.
\end{abstract}

\section{INTRODUCTION}

The many-valued logic paradigm extends binary logic to represent and manipulate partial truth: it is based on a symbolic, discrete truth scale and offers a formal framework to combine and reason with these degrees. Many variants have been proposed, differing in their interpretation, as well as their theoretical properties, see e.g. [1]-[3].

One of the reasons for the diversity of these variants in particular relates to the interpretation of the truth degree located at the middle position on the scale (usually, an odd number of intermediate truth levels is considered, so that the middle value is forced into the scale): as described and discussed in details in [4], it bears an intrinsic ambiguity. Indeed, on the one hand, it can be understood as a neutral truth value, neither true, nor false; on the other hand, it can be interpreted as expressing ignorance, i.e. the inability to assess the truth degree. As such, it corresponds to an information state of the agent, and not to a truth degree. It can thus be considered as representing partial truth or incomplete knowledge, although the two notions clearly have distinct nature [4].

The interpretation as ignorance opens the way to an epistemic reading, where the truth degrees should actually be understood as belief degrees. Therefore, it has been proposed to exploit modal logic to formulate Kleene three-valued logic and Belnap four-valued logic with improved characteristics [5].

This paper studies a different approach, based on an extension of many-valued logic, called $\mathcal{L}_{M}^{e}$ [6]: in this formalism, the distinction between neutral truth and ignorance is made through the introduction of an additional assessment value, that does not belong to the truth degree scale, as detailed in Section II-B: being outside the scale is the crucial feature that captures the essential difference between gradual truth and ignorance. This extended many-valued framework has been proposed to combine multi-criteria evaluations, so as to manipulate incomplete assessments of pieces of information [6].

In this paper a logical perspective is considered, to examine the properties of $\mathcal{L}_{M}^{e}$ as a reasoning framework, in a propositional logic case: the issue is to define appropriate operators to implement the semantics of the connectives, conjunction, disjunction and implication. A second task addresses the definition of an inference process, to allow to establish new pieces of knowledge together with their evaluation in $\mathcal{L}_{M}^{e}$, using an appropriate Modus Ponens variant. In both cases, the main question is how to manipulate ignorance.

The paper is structured as follows: after giving in Section II some preliminaries, regarding classic many-valued logic, $\mathcal{L}_{M}$, and its extension $\mathcal{L}_{M}^{e}$ to distinctly take into account ignorance, it studies in Section III the question of defining the semantics of the logical connectives to manipulate this ignorance. Section IV examines the issue of inference task in this framework. Section V concludes the paper.

\section{Preliminaries: $\mathcal{L}_{M}$ And $\mathcal{L}_{M}^{e}$}

This section briefly presents some of the main many-valued logic systems and discusses differences in their interpretations and their consequences on their definitions (see e.g. [1]-[3] for complete surveys and discussions). It then describes the extended many-valued framework on which the proposed tools to reason around ignorance rely.

\section{A. Many-Valued Logic: $\mathcal{L}_{M}$}

Many-valued logic models reasoning using a discrete truth scale allowing to represent and manipulate partial truth: it uses $M>2$ truth degrees of a totally ordered set $\mathcal{L}_{M}=$ $\left\{\tau_{0}, \ldots, \tau_{M-1}\right\}$, where $\tau_{\alpha} \leq \tau_{\beta} \Leftrightarrow \alpha \leq \beta$. The ordering property guarantees all degrees in $\mathcal{L}_{M}$ are comparable. They span, at a granularity varying with $M$, different levels of veracity from $\tau_{0}$, meaning 'false', to $\tau_{M-1}$, for 'true'. The switch between the two appears around the middle value $\tau_{\underline{M-1}}$, usually forced into the chosen scale by choosing an odd $\stackrel{M}{M}^{2}$.

This section briefly discusses some classic three-valued logics and then turns to their generalisations for higher $M$ values. Here as in the following sections, the logical symbols are abusively applied to truth degrees, and not only to logical formulae, to express the truth value of the compound formula for which the individual truth values are considered. 
TABLE I

TRUTH TABLES FOR SOME CLASSIC TRI-VALENT LOGICS

$\begin{array}{ccc}\text { Negation } & \text { Post } & \text { Others } \\ \neg \tau_{0} & \tau_{1} & \tau_{2} \\ \neg \tau_{1} & \tau_{2} & \tau_{1} \\ \neg \tau_{2} & \tau_{0} & \tau_{0}\end{array}$

Łukasiewicz $\mathrm{七}_{3}$

\begin{tabular}{c|ccc|ccc|ccc} 
& 0 & $\tau_{M-1}$ & 1 & 0 & $\tau_{\frac{M-1}{2}}$ & 1 & 0 & $\frac{\tau_{M-1}}{\tau^{2}}$ & 1 \\
& $\tau_{0}$ & $\tau_{1}$ & $\tau_{2}$ & $\tau_{0}$ & $\tau_{1}$ & $\tau_{2}$ & $\tau_{0}$ & $\tau_{1}$ & $\tau_{2}$ \\
\hline$\tau_{0}$ & $\tau_{0}$ & $\tau_{1}$ & $\tau_{2}$ & $\tau_{0}$ & $\tau_{0}$ & $\tau_{0}$ & $\tau_{2}$ & $\tau_{2}$ & $\tau_{2}$ \\
$\tau_{1}$ & $\tau_{1}$ & $\tau_{1}$ & $\tau_{2}$ & $\tau_{0}$ & $\tau_{1}$ & $\tau_{1}$ & $\tau_{1}$ & $\tau_{2}$ & $\tau_{2}$ \\
$\tau_{2}$ & $\tau_{2}$ & $\tau_{2}$ & $\tau_{2}$ & $\tau_{0}$ & $\tau_{1}$ & $\tau_{2}$ & $\tau_{0}$ & $\tau_{1}$ & $\tau_{2}$
\end{tabular}

\begin{tabular}{l|cll|lll|lll}
\multicolumn{2}{l}{ Bochvar $\mathrm{B}_{3}$} \\
\hline$\tau_{0}$ & $\tau_{0}$ & $\tau_{1}$ & $\tau_{2}$ & $\tau_{0}$ & $\tau_{1}$ & $\tau_{0}$ & $\tau_{2}$ & $\tau_{1}$ & $\tau_{2}$ \\
$\tau_{1}$ & $\tau_{1}$ & $\tau_{1}$ & $\tau_{1}$ & $\tau_{1}$ & $\tau_{1}$ & $\tau_{1}$ & $\tau_{1}$ & $\tau_{1}$ & $\tau_{1}$ \\
$\tau_{2}$ & $\tau_{2}$ & $\tau_{1}$ & $\tau_{2}$ & $\tau_{0}$ & $\tau_{1}$ & $\tau_{2}$ & $\tau_{0}$ & $\tau_{1}$ & $\tau_{2}$
\end{tabular}

\begin{tabular}{l|lll|lll|lll}
\multicolumn{1}{l}{ Kleene $\mathrm{K}_{3}$} \\
\hline$\tau_{0}$ & $\tau_{0}$ & $\tau_{1}$ & $\tau_{2}$ & $\tau_{0}$ & $\tau_{0}$ & $\tau_{0}$ & $\tau_{2}$ & $\tau_{2}$ & $\tau_{2}$
\end{tabular}

\begin{tabular}{llll|lll|lll}
$\tau_{1}$ & $\tau_{1}$ & $\tau_{1}$ & $\tau_{2}$ & $\tau_{0}$ & $\tau_{1}$ & $\tau_{1}$ & $\tau_{1}$ & $\tau_{1}$ & $\tau_{2}$
\end{tabular}

\begin{tabular}{c|ccc|ccc|ccc}
\multicolumn{10}{l}{ Post $\mathrm{P}_{3}$} \\
\hline$\tau_{0}$ & $\tau_{0}$ & $\tau_{1}$ & $\tau_{2}$ & $\tau_{1}$ & $\tau_{1}$ & $\tau_{1}$ & $\tau_{2}$ & $\tau_{2}$ & $\tau_{2}$ \\
$\tau_{1}$ & $\tau_{1}$ & $\tau_{1}$ & $\tau_{2}$ & $\tau_{1}$ & $\tau_{2}$ & $\tau_{0}$ & $\tau_{0}$ & $\tau_{1}$ & $\tau_{2}$ \\
$\tau_{2}$ & $\tau_{2}$ & $\tau_{2}$ & $\tau_{2}$ & $\tau_{1}$ & $\tau_{0}$ & $\tau_{0}$ & $\tau_{1}$ & $\tau_{1}$ & $\tau_{2}$
\end{tabular}

1) Original Tri-valued Logics: The first proposed manyvalued logic systems considered the case of three truth degrees, often denoted $0,1 / 2$ and 1 and mainly differ in the interpretation of the middle value. Their definitions are usually given through the truth table for the negation and one connective among $\{\vee, \wedge, \rightarrow\}$, the two other ones being derived from syntactical expressions. They also differ in the choice of the reference connective, its definition and the expressions to derive the others (see [1], [2]). Table I gives the resulting truth tables, using the notations introduced above i.e. denoting 0 as $\tau_{0}, 1 / 2$ as $\tau_{1}$ and 1 as $\tau_{2}$.

Post system is based on the definition of the truth tables for disjunction and negation, from which the other connectives are then derived. It is based on a very specific, cyclic, negation; as a consequence, it does not satisfy the normal conditions, i.e. it does not coincide with the classic binary logic case when restricted to the extreme degrees $\{0,1\}$, which makes it difficult to interpret.

The three other systems satisfy the normal conditions. The Bochvar system considers the intermediate value $\tau_{1}$ as an absorbing element, which makes any evaluation $\tau_{\alpha} \odot \tau_{\beta}=\tau_{1}$, for all operators $\odot \in\{\vee, \wedge, \rightarrow\}$ if $\tau_{\alpha}$ or $\tau_{\beta}$ equals $\tau_{1}$. Indeed, Bochvar considers that $\tau_{1}$ corresponds to ignorance, which "contaminates" any evaluation. As a consequence, $\tau_{0} \wedge \tau_{1} \neq \tau_{0}$ and $\tau_{2} \vee \tau_{0} \neq \tau_{2}$.

Łukasiewicz and Kleene systems return the intuitive values in these cases, they only differ in the evaluation of $\tau_{1} \rightarrow \tau_{1}$ : it is a tautology for $\mathrm{Ł}_{3}$ but not for $\mathrm{K}_{3}$. Indeed, $\mathrm{K}_{3}$ exploits a partial truth interpretation of the intermediate degree $\tau_{1}$ and considers that if both premise and conclusion are half true, so is the implication.
The issue of tautologies in tri-valent and general manyvalued logics is discussed in details in [1]. It has been proposed as a counter-argument to a truth table approach [4]. However it may be considered that the use of a general many-valued framework, that allows for several intermediary truth degrees between true and false, i.e. in the case where $M>3$, usually leads to abandon the principle of excluded middle (see e.g. [1] for a detailed discussion): it can thus be considered as relevant that some tautologies are not recovered.

2) General Case: Table II summarises six main manyvalued logics, as can be found for instance in [1], using the notations defined in the previous section. They are usually based on the definition of negation and a binary connective, as well as some syntactic equivalences which make it possible to derive the other binary connectives. The table omits a common alternative for implication, denoted $\tau_{\alpha} \supset \tau_{\beta}=\neg \tau_{\alpha} \vee \tau_{\beta}$, that is considered in the systems $U_{M}, S_{M}$ and $P_{M}$ and that leads to define $\tau_{\alpha} \supset \tau_{\beta}=\max \left(\neg \tau_{\alpha}, \tau_{\beta}\right)$ in all three cases.

In the case of $B_{M}$, two cases must actually be distinguished, depending on whether $M$ is odd or even, which determines whether $\tau_{\underline{M-1}}$ belongs to the scale or not. In the latter case, $\tau_{\underline{M-1}}$ must be replaced by $\tau_{\left\lfloor\frac{M-1}{2}\right\rfloor}$, i.e. the degree immediately below the intermediate level that does not belong to the scale. In Table II, both are denoted $\tau_{\underline{M-1}}$ to simplify the notations. The $U_{M}$ system only considers the case of odd $M$.

$\mathfrak{七}_{M}$ generalises the trivalent Łukasiewicz logic, $B_{M}$ the Bochvar logic, $S_{M}$ using the $\supset$ implication the Kleene logic, $G_{M}$ the Gödel logic and $P_{M}$ the Post logic [1].

Except $G_{M}$ and $P_{M}$, all systems agree on the negation definition. $G_{M}$ negation only allows two values as result, falling back to a binary logic. $P_{M}$ considers an atypical cyclic definition, generalising the one of $P_{3}$ indicated in Table I. It can be underlined that for all systems except $G_{M}$ and $P_{M}$, $\neg \tau_{\frac{M-1}{2}}=\tau_{\frac{M-1}{2}}$, for $G_{M}$, it equals $\tau_{0}$, i.e. false, and for $P_{M}$, $\tau_{\frac{M-1}{2}-1}$.

Except $B_{M}$, all systems agree on the definition of disjunction and conjunction, whether they are directly defined or derived from other connectives. In the case of $\succeq_{3}$, a so-called strong variant of these connectives is also proposed, according to which $\tau_{\alpha} \vee \tau_{\beta}=\neg \tau_{\alpha} \rightarrow \tau_{\beta}=\min \left(\tau_{\alpha+\beta}, \tau_{M-1}\right)$ and then $\tau_{\alpha} \wedge \tau_{\beta}=\max \left(\tau_{\alpha+\beta-(M-1)}, \tau_{0}\right)$. With this variant, $\tau_{\alpha} \vee \tau_{\alpha} \neq$ $\tau_{\alpha}$, except for $\tau_{\underline{M-1}}$, whereas it is the case for the consensus definition as $\max \left(\tau_{\alpha}^{2}, \tau_{\beta}\right)$. Regarding $B_{M}$, the disjunction has value $\tau_{\frac{M-1}{2}}$ except in the cases requiring compatibility with binary logic, i.e. when both $\tau_{\alpha}, \tau_{\beta} \in\left\{\tau_{0}, \tau_{M-1}\right\}$ : even more than in the tri-valued case, $\tau_{\frac{M-1}{2}}$ plays a pervasive role, $B_{M}$ actually reduces to $B_{3}$ after a few degree combination steps.

More variations are observed for the definition of the implication connective: all systems agrees on value $\tau_{M-1}$ if $\tau_{\alpha} \leq \tau_{\beta}$, but otherwise, the resulting value ranges from $\tau_{0}$ (for $S_{M}$ ) to $\tau_{\beta}$ (for $G_{M}$ ), or greater values depending on the difference between $\tau_{\alpha}$ and $\tau_{\beta}$.

\section{B. $\mathcal{L}_{M}^{e}$ : Introduction of an Ignorance Degree, $\tau_{\text {? }}$ ?}

It has been proposed to extend the previous many-valued framework, in order to distinguish explicitly between neutral 
TABLE II

OVERVIEW OF SIX CLASSIC MANY-VALUED LOGICS, AS CAN BE FOUND E.G. IN [1]

\begin{tabular}{|c|c|c|c|}
\hline & $Ł_{M}$ & $U_{M}$ & $B_{M}$ \\
\hline$\neg \tau_{\alpha}$ & $\tau_{M-1-\alpha}$ & $\tau_{M-1-\alpha}$ & $\tau_{M-1-\alpha}$ \\
\hline$\tau_{\alpha} \rightarrow \tau_{\beta}$ & $\begin{cases}\tau_{M-1} & \text { if } \tau_{\alpha} \leq \tau_{\beta} \\
\tau_{M-1-(\alpha-\beta)} & \text { otherwise }\end{cases}$ & $\begin{cases}\tau_{M-1} & \text { if } \tau_{\alpha} \leq \tau_{\beta} \\
\tau_{\frac{M-1}{2}} & \text { if } \tau_{\alpha} \geq \tau_{\beta} \\
& \text { and } \alpha-\beta \leq M-1 \\
\tau_{0} & \text { otherwise }\end{cases}$ & $\begin{cases}\tau_{M-1} & \text { if } \tau_{\alpha}=\tau_{0} \text { and } \tau_{\beta} \in\left\{\tau_{0}, \tau_{M-1}\right\} \\
\tau_{\beta} & \text { if } \tau_{\alpha}=\tau_{M-1} \text { and } \tau_{\beta} \in\left\{\tau_{0}, \tau_{M-1}\right\} \\
\frac{\tau_{M-1}}{2} & \text { otherwise }\end{cases}$ \\
\hline$\tau_{\alpha} \vee \tau_{\beta}$ & $\begin{array}{l}\left(\tau_{\alpha} \rightarrow \tau_{\beta}\right) \rightarrow \tau_{\beta} \\
\max \left(\tau_{\alpha}, \tau_{\beta}\right)\end{array}$ & $\begin{array}{l}\neg\left(\neg \tau_{\alpha} \wedge \neg \tau_{\beta}\right) \\
\max \left(\tau_{\alpha}, \tau_{\beta}\right)\end{array}$ & $\begin{array}{l}\neg\left(\neg \tau_{\alpha} \wedge \neg \tau_{\beta}\right) \\
\begin{cases}\max \left(\tau_{\alpha}, \tau_{\beta}\right) & \text { if } \tau_{\alpha}, \tau_{\beta} \in\left\{\tau_{0}, \tau_{M-1}\right\} \\
\frac{\tau_{M-1}}{2} & \text { otherwise }\end{cases} \end{array}$ \\
\hline$\tau_{\alpha} \wedge \tau_{\beta}$ & $\begin{array}{l}\neg\left(\neg \tau_{\alpha} \vee \neg \tau_{\beta}\right) \\
\min \left(\tau_{\alpha}, \tau_{\beta}\right)\end{array}$ & $\min \left(\tau_{\alpha}, \tau_{\beta}\right)$ & $\begin{cases}\min \left(\tau_{\alpha}, \tau_{\beta}\right) & \text { if } \tau_{\alpha}, \tau_{\beta} \in\left\{\tau_{0}, \tau_{M-1}\right\} \\
\frac{\tau_{M-1}}{2} & \text { otherwise }\end{cases}$ \\
\hline & $S_{M}$ & $G_{M}$ & $P_{M}$ \\
\hline$\neg \tau_{\alpha}$ & $\tau_{M-1-\alpha}$ & $\begin{array}{ll}\tau_{M-1} & \text { if } \tau_{\alpha}=\tau_{0} \\
\tau_{0} & \text { otherwise }\end{array}$ & $\begin{cases}\tau_{M-1} & \text { if } \tau_{\alpha}=\tau_{0} \\
\tau_{\alpha-1} & \text { otherwise }\end{cases}$ \\
\hline$\tau_{\alpha} \rightarrow \tau_{\beta}$ & $\begin{cases}\tau_{M-1} & \text { if } \tau_{\alpha} \leq \tau_{\beta} \\
\tau_{0} & \text { otherwise }\end{cases}$ & $\begin{cases}\tau_{M-1} & \text { if } \tau_{\alpha} \leq \tau_{\beta} \\
\tau_{\beta} & \text { otherwise }\end{cases}$ & $\begin{cases}\tau_{M-1} & \text { if } \tau_{\alpha}=\tau_{0} \\
\max \left(\tau_{\alpha-1}, \tau_{\beta}\right) & \text { otherwise }\end{cases}$ \\
\hline$\tau_{\alpha} \vee \tau_{\beta}$ & $\max \left(\tau_{\alpha}, \tau_{\beta}\right)$ & $\max \left(\tau_{\alpha}, \tau_{\beta}\right)$ & $\max \left(\tau_{\alpha}, \tau_{\beta}\right)$ \\
\hline$\tau_{\alpha} \wedge \tau_{\beta}$ & $\min \left(\tau_{\alpha}, \tau_{\beta}\right)$ & $\min \left(\tau_{\alpha}, \tau_{\beta}\right)$ & $\min \left(\tau_{\alpha}, \tau_{\beta}\right)$ \\
\hline
\end{tabular}

truth value and ignorance, and thus to remove the ambiguity between these two notions [6]: $\mathcal{L}_{M}^{e}$ introduces an additional value to assess a given formula, denoted $\tau_{?} . \mathcal{L}_{M}^{e}$ is not proposed as a reasoning framework, but as a framework to perform symbolic multi-criteria evaluation, more precisely for a task of information scoring: $\tau_{\text {? }}$ is primarily introduced to allow abstaining from evaluating some of the criteria and to make an explicit difference with the case where they have a neutral assessment.

As a consequence, $\tau_{\text {? }}$ is proposed to satisfy the following properties, where $\odot$ denotes a computational multivalued binary operator:

$$
\begin{array}{ll}
\left(P_{1}\right) & \tau_{?} \notin \mathcal{L}_{M} \\
\left(P_{2}\right) & \tau_{?} \odot \tau_{\alpha}=\tau_{\alpha} \odot \tau_{?}=\tau_{\alpha} \text { forall } \tau_{\alpha} \in \mathcal{L}_{M} \\
\left(P_{3}\right) & \neg \tau_{?}=\tau_{?} \\
\left(P_{4}\right) & \tau_{?} \odot \tau_{?}=\tau_{?}
\end{array}
$$

$\left(P_{1}\right)$ sets $\tau_{\text {? }}$ apart from other degrees, not submitting it to the same ordering constraints as $\tau_{\alpha} \in \mathcal{L}_{M}$ : an element which cannot be evaluated cannot be compared to an element whose evaluation is known, no matter its value. Therefore, $\tau_{\text {? }}$ is defined as an exception to the total order rule and of a different nature than other degrees.

$\left(P_{2}\right)$ defines $\tau_{\text {? }}$ as a neutral element for all considered manipulation operators: it applies a principle according to which what is not known should not be taken into account when combining the evaluation of several criteria. This can be interpreted as a neutral posture, for instance as opposed to an optimistic one that would replace the unknown by the highest possible degree. The aim of this paper is to study this principle in the case where logical operations are considered, i.e. in the case $\odot \in\{\wedge, \vee, \rightarrow\}$.

The last two properties $\left(P_{3}\right)$ and $\left(P_{4}\right)$ define the behaviour of $\tau_{\text {? }}$ when combined with itself.
The evaluation is then performed on $\mathcal{L}_{M}$ extended with $\tau_{\text {? }}$, denoted by $\mathcal{L}_{M}^{e}=\mathcal{L}_{M} \cup\left\{\tau_{\text {? }}\right\}$. It is shown to offer an essential gain in expressiveness, illustrated in an information scoring context.

\section{Logical CONNECTIVES IN $\mathcal{L}_{M}^{e}$}

The additional truth value $\tau_{\text {? }}$ has been introduced to manipulate the degrees at an arithmetical level [6], its reasoning properties have not been studied. This section examines extended semantics for the logical connectives, to study how they can process $\tau_{\text {? }}$. After discussing the cases of negation, disjunction and conjunction, the section details the case of implication, for which many choices can be considered. Of course, for the combination of $\tau_{\alpha}$ and $\tau_{\beta}$ in $\mathcal{L}_{M}$, i.e. distinct from $\tau_{\text {? }}$, classical operators as summarised in Table II can be used. The issue is to define their combination with $\tau_{\text {? }}$, as well as its combination with itself.

\section{A. Negation, Disjunction and Conjunction Semantics in $\mathcal{L}_{M}^{e}$}

This section discusses the cases of the negation, disjunction and conjunction connectives in turn.

1) Negation Connective: We propose to set

$$
\begin{aligned}
\neg \tau_{\alpha} & =\tau_{M-1-\alpha} \text { if } \tau_{\alpha} \in \mathcal{L}_{M} \\
\neg \tau_{?} & =\tau_{?}
\end{aligned}
$$

The first line agrees with most classical negation (see Table II), the second one is identical to property $\left(P_{3}\right)$. They correspond to a classic choice for $\mathcal{L}_{M}$ and an arguably natural one for $\tau_{?}$ : there does not seem to be a reason why the negation of ignorance should be made to a known value, nor how one should be selected.

This definition leads to a similarity in behaviour between $\tau_{\text {? }}$ and $\tau_{\frac{M-1}{2}}$ : they are both equal to their own negation. 
2) Disjunction Connective: As for instance discussed in [2], and using the notations introduced in Section II, a disjunction operator is required to satisfy the properties of associativity, commutativity, non-decreasing monotonicity in both arguments and to have $\tau_{0}$ as neutral element.

In the case of $\mathcal{L}_{M}^{e}$ however, two of these properties need to be questioned: first, as the set of truth values is not ordered, by definition (property $\left(P_{1}\right)$ ), the monotonicity requirement cannot be defined and thus will not apply. Second, the definition of $\tau_{0}$ as neutral element is also reconsidered.

Indeed, as mentioned in Section II-B, an underlying principle of $\mathcal{L}_{M}^{e}$ states that what is not known should not be taken into account. It leads to set, for all $\tau_{\alpha} \in \mathcal{L}_{M}^{e}$,

$$
\tau_{?} \vee \tau_{\alpha}=\tau_{\alpha}
$$

considering that, in absence of knowledge, the disjunction is as true as its known component, in agreement with property $\left(P_{2}\right)$.

This equation leads to $\tau_{\text {? }} \vee \tau_{\text {? }}=\tau_{\text {? }}$ which seems to be a natural choice. Note that as a consequence of the negation definition, it also leads to consider that $\neg \tau_{\text {? }} \vee \tau_{\text {? }}=\tau_{\text {? }}$, which may be debatable [4]. However, as mentioned before, manyvalued logic does not require the excluded middle to hold, so it can be argued that it should not be required for the specific case of $\tau_{\text {? }}$ either.

Another consequence of this choice is that $\tau_{0} \vee \tau_{\text {? }}=\tau_{0}$, meaning that $\tau_{0}$ is not a neutral element. However, it still holds that $\tau_{M-1}$ is an absorbing element: for $\tau_{\alpha} \in \mathcal{L}_{M}$, the proposed definition does not modify the behaviour, therefore $\tau_{M-1} \vee \tau_{\alpha}=\tau_{M-1}$, and for $\tau_{\text {? }}$, it holds that $\tau_{M-1} \vee \tau_{\text {? }}=$ $\tau_{M-1}$.

This behaviour defined for $\tau_{\text {? }}$ differs from the one for $\tau_{\frac{M-1}{2}}$ : in most cases (see Table II), it holds that $\tau_{\frac{M-1}{2}} \vee \tau_{\alpha}={ }^{2} \tau_{\alpha}$ only for $\tau_{\alpha} \geq \tau_{\frac{M-1}{2}}$, otherwise $\tau_{\frac{M-1}{2}} \vee \tau_{\alpha} \stackrel{\frac{2}{=}}{=} \tau_{\frac{M-1}{2}}: \tau_{\text {? }}$ is a neutral element for disjunction, whereas $\frac{\tau_{\frac{M-1}{2}}}{2}$ only is for greater values, it is absorbing otherwise.

3) Conjunction Connective: The definition of conjunction semantics can be derived from that of the disjunction and negation (Eq. (1) and (2)), based on the De Morgan laws: $\tau_{\alpha} \wedge \tau_{\beta}=\neg\left(\neg \tau_{\alpha} \vee \neg \tau_{\beta}\right)$ and, therefore, $\tau_{\alpha} \wedge \tau_{\text {? }}=\neg\left(\neg \tau_{\alpha} \vee\right.$ $\left.\tau_{\text {? }}\right)=\neg\left(\neg \tau_{\alpha}\right)=\tau_{\alpha}$ : as a consequence

$$
\tau_{?} \wedge \tau_{\alpha}=\tau_{\alpha}
$$

Another approach to define conjunction can be based on a discussion similar to the one conducted for the disjunction in the previous subsection. It leads to an identical result as the one derived using the De Morgan laws.

Usually, a conjunction operator is rquired to satisfy the properties of associativity, commutativity, non-decreasing monotonicity and to have $\tau_{M-1}$ as neutral element (see e.g. [2]): as for disjunction, monotonicity and neutral element do not hold in $\mathcal{L}_{M}^{e}$, however, $\tau_{0}$ remains an absorbing element.

\section{B. Implication Semantics in $\mathcal{L}_{M}^{e}$}

The implication definition opens the way to more discussions: there is no general agreement on the properties an implication operator should satisfy (see e.g. [2] and [7]) and numerous derivations from disjunction, conjunction and negation can be considered (see for instance [2]). They are discussed in turn below.

1) Residuated Implications: R-implications are derived from conjunction and defined as $\tau_{\alpha} \rightarrow \tau_{\beta}=\sup \left\{\tau \mid \tau_{\alpha} \wedge \tau \leq\right.$ $\left.\tau_{\beta}\right\}$. As a consequence, this approach cannot be applied in the $\mathcal{L}_{M}^{e}$ case: it requires a total ordering on the considered truth values, whereas the essential characteristic of $\mathcal{L}_{M}^{e}$ precisely is its absence of total order, that makes it possible to introduce the crucial difference between $\tau_{\text {? }}$ and any truth degree $\tau_{\alpha}$.

More precisely, due to the conjunction operator (Eq. 3), if $\tau_{\alpha}=\tau_{\text {? }}$, the r-implication definition can lead to set $\tau_{\text {? }} \rightarrow \tau_{\beta}=$ $\sup \left\{\tau \mid \tau_{?} \wedge \tau \leq \tau_{\beta}\right\}=\sup \left\{\tau \mid \tau \leq \tau_{\beta}\right\}=\tau_{\beta}$. However, the value for $\tau_{\alpha} \rightarrow \tau_{\text {? }}$ cannot be established using this definition, nor that of $\tau_{?} \rightarrow \tau_{\text {? }}$.

2) Strong Implications: S-implications are derived from negation and disjunction as $\tau_{\alpha} \rightarrow \tau_{\beta}=\neg \tau_{\alpha} \vee \tau_{\beta}$. Using the definitions of Eq. (1) and (2), if $\tau_{\alpha}$ and $\tau_{\beta}$ denote values different from $\tau_{\text {? }}$ (i.e. $\tau_{\alpha}$ and $\tau_{\beta} \in \mathcal{L}_{M}$ ), it leads to

$$
\begin{aligned}
& \tau_{?} \rightarrow \tau_{\beta}=\tau_{\beta} \\
& \tau_{\alpha} \rightarrow \tau_{?}=\neg \tau_{\alpha} \\
& \tau_{?} \rightarrow \tau_{?}=\tau_{?}
\end{aligned}
$$

For $\tau_{\text {? }} \rightarrow \tau_{\beta}$, this definition leads to the same value as the one suggested by r-implications. However, it also allows to process the other cases. Setting $\tau_{\text {? }}$ for the case $\tau_{\text {? }} \rightarrow \tau_{\text {? }}$ is a natural choice, and is in agreement with property $\left(P_{4}\right)$. The specificity comes from $\tau_{\alpha} \rightarrow \tau_{\text {? }}=\neg \tau_{\alpha} \vee \tau_{\text {? }}=\neg \tau_{\alpha}$ because of the disjunction definition given in Eq. (2).

This choice allows to make the difference between $\tau_{\text {? }}$ and $\tau_{\frac{M-1}{2}}$ : depending on the values of $\tau_{\alpha}$ and $\tau_{\beta}$, neither does $\tau_{\frac{M-1}{2}} \rightarrow \tau_{\beta}$ always equal $\tau_{\beta}$, nor does $\tau_{\alpha} \rightarrow \tau_{\frac{M-1}{2}}$ always equal $\neg \tau_{\alpha}$. Thus there are cases where the result differs between $\tau_{\text {? }}$ and $\tau_{\underline{M-1}}$.

3) Quantum Logic Implications: QL-implications are derived from conjunction and negation as $\tau_{\alpha} \rightarrow \tau_{\beta}=\neg\left(\tau_{\alpha} \wedge\right.$ $\left.\neg\left(\tau_{\alpha} \wedge \tau_{\beta}\right)\right)$ Using the definitions given in Eq. (1) and (3), if $\tau_{\alpha}$ and $\tau_{\beta}$ denote values different from $\tau_{\text {? }}$, they lead to

$$
\begin{array}{lccc}
\tau_{?} \rightarrow \tau_{\beta} & = & \tau_{\beta} \\
\tau_{\alpha} \rightarrow \tau_{?} & = & \neg \tau_{\alpha} \vee \tau_{\alpha} \\
\tau_{?} \rightarrow \tau_{?} & = & \tau_{?}
\end{array}
$$

This approach agrees with the previous ones for $\tau_{?} \rightarrow \tau_{\beta}$ and $\tau_{\text {? }} \rightarrow \tau_{\text {? }}$, the difference comes from the $\tau_{\alpha} \rightarrow \tau_{\text {? }}$, whose result depends on the choice of the disjunction for values in $\mathcal{L}_{M}$ : it can be a tautology, i.e. $\tau_{M-1}$, if the law of excluded middle is imposed, or $\tau_{\max (\alpha, M-1-\alpha)}$ using classic variants (see Table II). As s-implications, it leads to different behaviours for $\tau_{\frac{M-1}{2}}$ and $\tau_{\text {? }}$.

4) Proposed Implication: We propose another variant for implication with ignorance, which can be considered as a radical choice that always produces $\tau_{\text {? }}$, in agreement with $\left(P_{2}\right)$ :

$$
\begin{aligned}
& \tau_{?} \rightarrow \tau_{\beta}=\tau_{?} \\
& \tau_{\alpha} \rightarrow \tau_{?}=\tau_{?} \\
& \tau_{?} \rightarrow \tau_{?}=\tau_{?}
\end{aligned}
$$


This choice relies on the principle that if the premise or the conclusion is unknown, it is not relevant to try to make a guess, and to try for instance a cautious or, on the contrary, an optimistic choice for the unknown value: the strong implication can be interpreted as using the minimal value that can be established, in a cautious approach. Indeed, for instance for the case $\tau_{\alpha} \rightarrow \tau_{\text {? }}$, if the conclusion turns out to be $\tau_{0}$, the truth value of the implication would be $\tau_{\alpha}$, whereas it would be $\tau_{M-1}$ if the conclusion turns out to be $\tau_{M-1}$. Setting the result as $\tau_{\alpha}$ thus corresponds to the most cautious choice. The same interpretation applies to the case $\tau_{\text {? }} \rightarrow \tau_{\beta}$.

The proposed implication rather keeps the unknown state and propagates it: if either the premise or the conclusion cannot be evaluated, it is considered that the truth value of the implication cannot be evaluated either. The next section examines the consequence of this choice on the inference process to derive new pieces of information.

Obviously, this choice it leads to different behaviours for $\tau_{\frac{M-1}{2}}$ and $\tau_{?}$.

\section{Consistency Issue}

The consistency issue of a logical system is related to its impossibility to infer a contradiction. In the case of manyvalued logic, this property is already relaxed since the laws of excluded middle and noncontradiction usually do not hold.

Now adding $\tau_{\text {? }}$ does not introduce inconsistency: for disjunction and conjunction, $\tau_{\text {? }}$ is defined as a neutral element, for the proposed implication it is a absorbing element. As a consequence, any inconsistency proved after its inclusion will persist should it be removed.

\section{LOGICAL INFERENCE IN $\mathcal{L}_{M}^{e}$}

The inference task aims at establishing new pieces of knowledge, i.e. new formulae that can be derived from given formulae, together with their truth values. After discussing the considered issue, this section describes the proposed inference scheme based on an extended Modus Ponens rule and illustrates it in the case of $\mathcal{L}_{5}^{e}$.

\section{A. Principle and Discussion}

Inference in many-valued logic (see e.g. [2]) usually is a binary question, based on the notion of designated truth values, i.e. a set of truth degrees that are interpreted as degrees of truth, as opposed to anti-designated values, interpreted as degrees of falsity: inference requires that if a set of formulae has designated truth values, then a derived formula also has a designated truth value.

The issue considered here is closer to the inference question in fuzzy logic, where the aim is to precisely identify the truth value of the derived formula: formally, given a set of propositional formulae associated with their truth values $\mathcal{F}=$ $\left\{\left(F_{i}, \tau^{i}\right), i=1 . . n\right\}$, where $F_{i}$ are formulae and $\tau^{i} \in \mathcal{L}_{M}^{e}$, the question is to examine what can be established for a given formula $G$ regarding its truth value. It is thus related to the issue of tableau calculus for many-valued logic (see e.g. [2] for an overview).
The next section discusses the case of Modus Ponens, also called rule of detachment, i.e. it considers $\mathcal{F}=$ $\left\{\left(F, \tau^{F}\right),\left(F \rightarrow G, \tau^{I}\right)\right\}$, where $F$ and $G$ can be compound complex formulae, and when $\tau^{F}, \tau^{I} \in \mathcal{L}_{M}^{e}$ : the question is to possibly infer $\left(G, \tau^{G}\right)$, establishing the value of $\tau^{G}$.

\section{B. Extended Modus Ponens}

In binary logic, the Modus Ponens inference rule allows to infer $G$ from $\mathcal{F}=\{F, F \rightarrow G\}$ as the single interpretation that makes both the observation $F$ and the rule $F \rightarrow G$ true also makes $G$ true. In fuzzy logic, for instance considering the Łukasiewicz operators, $\mathcal{F}=\{(F, u),(F \rightarrow G, v)\}$ where $u, v \in[0,1]$, the Generalised Modus Ponens derives $(G, \max (0, u+v-1))$.

In the case of propositional $\mathcal{L}_{M}^{e}$, we propose to examine all interpretations compatible with $\mathcal{F}$ i.e. the set $\mathcal{T}=$ $\left\{\tau \mid i\left(\tau^{F}, \tau\right)=\tau^{I}\right\}$ where $i$ is the proposed implication operator discussed in the previous section. The definition of $\tau^{G}$ depends on the cardinality of $\mathcal{T}$ and distinguishes between three cases:

1) if $|\mathcal{T}|=1$ : i.e. if a single interpretation is compatible, then the truth value of $G$ naturally equals this value.

2) if $|\mathcal{T}|>1$ : i.e. if several interpretations are possible, several approaches can be considered: one consists in abstaining from taking a decision and allowing truth values as subsets of $\mathcal{L}_{M}^{e}$. This approach can be related to the manyvalued tableau calculus, but may be difficult to interpret.

Another approach consists in aggregating the obtained values: a cautious choice can for instance select the minimal value, i.e. setting $\tau^{G}=\min \{\tau \in \mathcal{T}\}$, indicating that at least this degree can be achieved. This definition makes sense under the assumption that $\mathcal{T}$ does not contain both $\tau_{\text {? }}$ and values from $\mathcal{L}_{M}$, otherwise the min operator cannot be applied. Now as illustrated in the next section, when using the proposed implication defined in Eq. (6), the only problematic case is obtained if $\tau^{F}=\tau^{I}=\tau_{?}$ : in such a case, it seems reasonable to set that $\tau^{G}=\tau$ ?

A third approach can also consider that if several values can be obtained, it means that the available pieces of information are not conclusive enough, i.e. that the rule together with the observation does not allow to conclude: this principle leads to set $\tau^{G}=\tau_{\text {? }}$ in such a case.

3) if $\mathcal{T}=\emptyset$ : i.e. no interpretation is compatible with $\mathcal{F}$, then we propose to set $\tau^{G}=\tau_{\text {? }}$, meaning that the rule does not allow to infer any new piece of information.

It may be argued that this case corresponds to a contradiction and requires to be processed as such. However the expressiveness allowed by the introduction of $\tau$ ? allows to take a less definite decision. Indeed, if $\tau^{G}=\tau_{\text {? }}$, using the proposed implication defined in Eq. (6), the truth value of the implication $F \rightarrow G$ gets value $\tau \rightarrow \tau_{\text {? }}=\tau_{\text {? }}$ which is not contradictory with the available value $\tau^{I}$ as $\tau^{I} \wedge \tau_{\text {? }}=\tau^{I}$ due to the conjunction operator defined in Eq. (3).

\section{Illustration in $\mathcal{L}_{5}^{e}$}

This section illustrates the proposed extended Modus Ponens in the case of $\mathcal{L}_{5}^{e}$, considering for implication operator 
TABLE III

TRUTH TABLE OF THE PROPOSED INFERENCE SCHEME, BASED ON EQ. (6) AND THE ŁUKASIEWICZ IMPLICATION.

\begin{tabular}{ccc|ccccc} 
& & \multicolumn{7}{|c}{$\tau^{I}$} \\
& & $\tau_{0}$ & $\tau_{1}$ & $\tau_{2}$ & $\tau_{3}$ & $\tau_{4}$ & $\tau_{?}$ \\
\hline \multirow{3}{*}{$\tau^{F}$} & $\tau_{0}$ & $\tau_{?}$ & $\tau_{?}$ & $\tau_{?}$ & $\tau_{?}$ & $\tau_{0}$ & $\tau_{?}$ \\
& $\tau_{1}$ & $\tau_{?}$ & $\tau_{?}$ & $\tau_{?}$ & $\tau_{0}$ & $\tau_{1}$ & $\tau_{?}$ \\
& $\tau_{2}$ & $\tau_{?}$ & $\tau_{?}$ & $\tau_{0}$ & $\tau_{1}$ & $\tau_{2}$ & $\tau_{?}$ \\
& $\tau_{3}$ & $\tau_{?}$ & $\tau_{0}$ & $\tau_{1}$ & $\tau_{2}$ & $\tau_{3}$ & $\tau_{?}$ \\
& $\tau_{4}$ & $\tau_{0}$ & $\tau_{1}$ & $\tau_{2}$ & $\tau_{3}$ & $\tau_{4}$ & $\tau_{?}$ \\
& $\tau_{?}$ & $\tau_{?}$ & $\tau_{?}$ & $\tau_{?}$ & $\tau_{?}$ & $\tau_{?}$ & $\tau_{?}$
\end{tabular}

TABLE IV

TRUTH TABLE OF THE ŁUKASIEWICZ GMP INFERENCE SCHEME.

\begin{tabular}{cc|ccccc} 
& & & & $\tau^{I}$ & & \\
& & & & & & \\
$\tau_{0} F$ & $\tau_{1}$ & $\tau_{2}$ & $\tau_{3}$ & $\tau_{4}$ \\
\hline & $\tau_{0}$ & $\tau_{0}$ & $\tau_{0}$ & $\tau_{0}$ & $\tau_{0}$ & $\tau_{0}$ \\
& $\tau_{1}$ & $\tau_{0}$ & $\tau_{0}$ & $\tau_{0}$ & $\tau_{0}$ & $\tau_{1}$ \\
& $\tau_{2}$ & $\tau_{0}$ & $\tau_{0}$ & $\tau_{0}$ & $\tau_{1}$ & $\tau_{2}$ \\
& $\tau_{3}$ & $\tau_{0}$ & $\tau_{0}$ & $\tau_{1}$ & $\tau_{2}$ & $\tau_{3}$ \\
& $\tau_{4}$ & $\tau_{0}$ & $\tau_{1}$ & $\tau_{2}$ & $\tau_{3}$ & $\tau_{4}$
\end{tabular}

the proposed operator defined in Eq. (6) combined with the Łukasiewicz operator for values other than $\tau_{\text {? }}$ (see first column of Table II). Table III gives the obtained results, where the colours give details about the computations: blue values correspond to the case where $\mathcal{T}=\emptyset$ and green ones to the case where $|\mathcal{T}|>1$, using the min operator as aggregation. Table IV gives the result obtained when applying a fuzzylike Generalized Modus Ponens (as mentioned in the previous section) to the discrete case of $\mathcal{L}_{5}$, as a comparison.

In the case where it is not known whether the rule $F \rightarrow G$ holds, i.e. when $\tau^{I}=\tau_{\text {? }}$ (see last column in Table III), it seems reasonable that the extended Modus Ponens leads to the conclusion that $\tau^{G}=\tau_{\text {? }}$, whatever the truth value of $F$ is: indeed, no information can be inferred from an unknown rule. This desired result is recovered due to the fact that if $\tau^{F} \neq \tau_{\text {? }}$, the only way to obtain $\tau_{\text {? }}$ for the implication $F \rightarrow G$ comes from the case where $\tau^{G}=\tau_{\text {? }}$. If $\tau^{F}=\tau_{\text {? }}$, actually $\mathcal{T}=\mathcal{L}_{5}^{e}$ and thus contains both contains $\tau_{\text {? }}$ and truth degrees. However it seems reasonable to set $\tau^{G}=\tau_{\text {? }}$ in this case.

When it is not known whether the observation $F$ holds, i.e. when $\tau^{F}=\tau_{\text {? }}$ (see last row in Table III), then whatever the truth value of the rule, again, as can be desired, the truth value of the conclusion is $\tau_{\text {? }}$. However, the justification for these values differs from the previous ones: they come from the fact that then $\mathcal{T}=\emptyset$.

All the previous cases correspond to cases where, indeed, $F$ and $F \rightarrow G$ alone do not justify deriving the conclusion $G$, making the resulting value $\tau_{\text {? }}$ relevant. Note that it may be the case that $G$ holds, due to other inferences or as initial piece of knowledge, but the derivation through $F$ and $F \rightarrow G$ is not conclusive. However, if a known truth degree $\tau$ can be established from a different inference, the combination will lead to $\tau \vee \tau_{\text {? }}=\tau$, preserving the identified piece of information. This also justifies the choice of the semantics for the disjunction connective discussed in the previous section.

Other cases where the result is $\tau_{\text {? }}$ can be observed in the upper left part of Table III: again, they can be interpreted as cases where the observation together with the rule are not conclusive, because either one or the other have too low truth degrees. This behaviour differs from the GMP one (see Table IV), where the conclusion is considered to be false, as $\tau^{G}=\tau_{0}$. The choice of not triggering the rule and getting $\tau_{\text {? }}$ ? as a result can be considered as relevant, even if debatable.

The rest of the two tables is identical, in particular thanks to the considered min aggregation for the blue values. It can be argued that the fact that a conclusion is considered false ( $\left.\tau^{G}=\tau_{0}\right)$ if both the rule and the observation are half true $\left(\tau_{\frac{M-1}{2}}=\tau_{2}\right)$ is not intuitive, but this is a result of building here the inference scheme on the $t_{5}$ implication operator, for which $\tau_{2} \rightarrow \tau_{0}=\tau_{2}$ : other implication choices can lead to other results, although the whole table needs to be recomputed and interpreted.

If the observation is true $\left(\tau^{F}=\tau_{4}\right)$ and the rule is half true $\left(\tau^{I}=\tau_{2}\right)$, then the conclusion is considered to be half true, whereas it is unknown, $\tau_{\text {? }}$, if the rule also is: beside allowing to avoid triggering the rule in inappropriate cases, as discussed above, the introduction of $\tau_{\text {? }}$ also allows to distinguish between ignorance and neutral value, in agreement with its initial motivations.

\section{COnClusion And Future Works}

This paper discussed, from a logical point of view, the introduction of an additional truth value, distinct from a truth degree, in the framework of many-valued logic, so as to enable to explicitly distinguish between neutral truth and ignorance: $\tau_{\text {? }}$ places the comparison between a 'half true' fact and another back in its rightful place in $\mathcal{L}_{M}$ 's semantic hierarchy. The definition of appropriate connective semantics has been discussed, illustrating alternative possibilities in particular for implication. For a specific choice, the issue of inference through an extended Modus Ponens has also been discussed, highlighting how $\tau_{\text {? }}$ allows to increase the legibility and expressiveness of the induced reasoning framework through the allowed disambiguisation. Ongoing works aim at further studying $\mathcal{L}_{M}^{e}$ at a theoretical level, in particular examining other inference schemes, as well as at an applicative level, to assess the practical relevance of the proposed tools for a real-life approximate reasoning framework.

\section{REFERENCES}

[1] N. Rescher, Many-valued Logic. McGraw Hill, 1969.

[2] S. Gottwald, A treatise on many-valued logics, ser. Studies in logic and computation. Baldock: Research Studies Press, 2001, vol. 9.

[3] - "Many-valued logics," in Handbook of the Philosophy of Sciences, D. Jacquette, Ed. Elsevier, 2007, pp. 675-722.

[4] D. Dubois, "On ignorance and contradiction considered as truth-values," Logic Journal of the IGPL, vol. 16, no. 2, pp. 195-216, 2008.

[5] — "Reasoning about ignorance and contradiction: many-valued logics versus epistemic logic," Soft Computing, vol. 16, no. 11, pp. 1817-1831, 2012.

[6] A. Revault d'Allonnes and M.-J. Lesot, "Formalising information scoring in a multivalued logic framework," in Proc. of IPMU'14, vol. 1, no. CCIS442. Springer, 2014, pp. 314-323.

[7] R. Yager, "On some new classes of implication operators and their role in approximate reasoning," Information Sciences, vol. 167, pp. 193-216, 2004. 